\title{
LoRaWAN for city scale IoT deployments
}

\author{
Philip J. Basford, Steven J. Johnston*, Mihaela Apetroaie-Cristea, Florentin M. J. Bulot and Simon J. Cox \\ Faculty of Engineering and Physical Sciences, University of Southampton, UK. \\ *sjj698@zepler.org
}

\begin{abstract}
LoRaWAN is a Low-Power Wide Area Network (LPWAN) technology designed for Internet of Things (IoT) deployments, this paper presents experiences gained from deploying a citywide LoRaWAN network across Southampton, UK. This network has been deployed to support an installation of air quality sensors and to explore the LoRaWAN technology. This deployment uses a mixture of commercial off-the-shelf gateways and custom gateways. The custom gateways have required the development of a new printed circuit board interface which is available open-source. The gateway locations have been chosen based on combination of availability and predicted coverage. This paper shows that this approach has provided a reasonable coverage of the city.
\end{abstract}

Index Terms-Internet of Things, Wireless sensor networks, Communication networks.

\section{INTRODUCTION}

Smart city deployments of Internet of Things (IoT) technologies are driving innovations and research in long range low power wireless communication networks. Previous Wireless Sensor Network (WSN) deployments would have used custom protocols to facilitate communication. The developments in this area have led to a new type of wireless communication networks, Low-Power Wide Area Networks (LPWANs). These technologies include: LoRaWAN [1], Sigfox [2], and NBIoT [3], which have reduced the complexity of developing a new IoT device. The city of Southampton, UK is used as a test bed to evaluate one of these LPWAN technologies: LoRaWAN. This evaluation has required deploying the necessary gateway infrastructure, as well as assessing its performance. The challenges of deploying gateway infrastructure vary depending on the desired scale. Deploying an LPWAN network to give city wide coverage poses significantly more challenges than setting up a simple test network. This LoRaWAN network has not been setup in isolation, it is used as communication means for the air quality monitors which are currently being deployed in and around Southampton [4], [5]. A comparison between different LPWAN technologies is presented in Section II, further details of the use case for air quality are given in Section III. The hardware in use and its applicability for different deployment scenarios are given in Section IV, and analysis of the gateways deployed presented in Section V, the paper concludes in Section VI.

\section{Low-Power Wide Area Network (LPWAN)}

Bardyn et al. state the main characteristics of a LPWAN are: ultra low-power operation, low cost, no need to wake an end device to maintain network connectivity, easy to deploy infrastructure nationwide, secure data transfer [7]. While not included in this list, long range is also a defining feature of these networks. This means that these LPWAN technologies are not competitors to Bluetooth, WiFi, Zigbee or other short range wireless communication technologies. A detailed comparison between LoRaWAN, Sigfox and NB-IoT is presented by Mekki et al. [6], and summarised in Table I. Despite NB-IoT using licensed frequencies compared to LoRaWAN and Sigfox which use the license free Industrial, Scientific and Medical (ISM) band all technologies have the same problem that the frequencies available in each region differ. This regulatory complexity creates additional difficulties when moving devices internationally. All three networks also offer encryption of the payload to prevent casual eavesdropping of traffic. Security analysis of the LoRaWAN protocol found multiple weaknesses in the LoRaWAN V1.0 specification [8] many of which have been addressed in version 1.1 [9], which is available but has yet to be adopted.

LoRaWAN is the only network for which it is easy and simple to deploy your own gateway. Both Sigfox and NB-IoT are operated by infrastructure companies and any additional gateway has to fit within the national operators deployment plan. A personal Sigfox gateway has recently been announced but distribution is managed by the local network operators who have to be contacted for information [10]. There are multiple vendors offering pre-built LoRaWAN gateways for sale, as well as instructions to make your own gateway from a kit of parts. The ability to build and/or deploy your own LoRaWAN gateway means that it is possible to run your own network entirely separate from others. This ability to create separate networks may be beneficial in commercial or defence market segments, but increased coverage is available by connecting to the community public network.

This community is based around The Things Network (TTN) [11]. TTN currently has $\approx 60000$ members, providing $\approx 6000$ gateways across $\approx 140$ countries. Data received by TTN is made available to the device owner as an MQTT topic which can then be processed by the owner however they wish. TTN handles the de-duplication of messages that have been received by multiple gateways simultaneously further reducing the complexity of implementation.

Localisation of devices based on multilateration of signals is a well established technique [12]. Both Sigfox and LoRaWAN offer support for localisation using different methods. LoRaWAN supports both Timed Difference of Arrival (TDOA) and Received Signal Strength Indication (RSSI) for multilateration of transmissions. This enables devices without Global Navigation Satellite System (GNSS) receivers to pro- 
TABLE I

COMPARISON OF DIFFERENT LOW-POWER WIDE AREA NeTWORK (LPWAN) TECHNOLOGIES [6]. All TECHNOLOGIES LISTED SUPPORT BI-DIRECTIONAL COMMUNICATION, THE STANDARD FOR EACH TECHNOLOGY IS DRIVEN BY DIFFERENT ORGANISATIONS. THE DIFFERENT

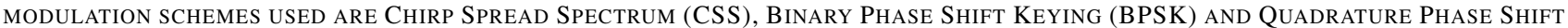
KEYING (QPSK), THE DIFFERENT LOCALISATION SCHEMES USED ARE RECEIVED SIGNAL STRENGTH INDICATION (RSSI) AND TIMED DifFERENCE OF ARRIVAL (TDOA). ADAPTED FROM [5].

\begin{tabular}{lccc} 
& LoRaWAN & Sigfox & NB-IoT \\
\hline Frequency bands & Unlicensed & Unlicensed & Licensed \\
Range (urban) & $5 \mathrm{~km}$ & $10 \mathrm{~km}$ & $1 \mathrm{~km}$ \\
Range (rural) & $20 \mathrm{~km}$ & $40 \mathrm{~km}$ & $10 \mathrm{~km}$ \\
Maximum data rate & $50 \mathrm{kbit} / \mathrm{s}$ & $0.1 \mathrm{kbit} / \mathrm{s}$ & $200 \mathrm{kbit} / \mathrm{s}$ \\
Maximum messages per day & Unlimited & $140 \mathrm{Up}, 4$ Down & Unlimited \\
Modulation & CSS & BPSK & QPSK \\
Encryption & Yes & No & Yes \\
Adaptive Data Rate (ADR) & Yes & No & No \\
Private networks & Yes & No & No \\
Gateways locations determined by & Anyone & Operator & Operator \\
Localisation & RSSI \& TDOA & RSSI & No
\end{tabular}

vide location aware data streams.

The ability to deploy your own LoRaWAN gateways, the greater traffic allowances, better localisation options and the strength of the community developing with LoRaWAN made it the first choice for this research into city scale IoT deployments. Previous work using LoRa for a smart city environment concluded that more robust communication could be achieved by using LoRaWAN on the LoRa physical layer [13].

\section{THE USE CASE}

The deployed LoRaWAN network has two primary purposes: providing data connectivity for an on-going air quality monitoring project and evaluating LoRaWAN for city-scale IoT deployments. Air pollution is a major influence on worldwide health, with 6.5 million premature deaths associated with air pollution in 2015 [14]. Southampton, shown in Figure 1, is a city with a population of $\approx 250000$ [15]. The city is situated on the South coast of England, and is surrounded by two motorways, has an airport, commercial dock and cruise ship terminal. It is an area with multiple sources of particulate matter pollution, and air quality is a major concern. To obtain the spatial resolution needed to assess personal exposure to pollution multiple sensors need to be deployed around the city. These deployment locations may not have network connections available, in which case LoRaWAN can be used for data transfer.

A further use case for the LoRaWAN network is by university students for their undergraduate projects and coursework. So as to not restrict the potential areas for deployment and testing a citywide coverage is needed.

\section{LORAWAN HARDWARE}

When using LoRaWAN networks there are two categories of device, each with different requirements: end nodes and gateways. The end nodes are designed to be primarily transmit nodes, and may have no other external connections. The gateways are primarily receivers, and as such have to be listening continuously to ensure that packets are not missed due to downtime. This difference in behaviour leads to the power demands of gateway nodes being much greater than end nodes. The gateways also require an uplink connection to transmit the packets onwards, this typically takes the form of either a wired/WiFi network connection, or a Global System for Mobile communications (GSM) data link. Gateways using a satellite uplink are also available. The use of satellites to receive LoRaWAN traffic directly is under development [18] and while this technology is not currently used in this deployment it is of interest for future rural deployments.

\section{A. End nodes}

Through the course of the work multiple end devices have been used. Three main devices have been used by the authors of this paper to generate LoRaWAN traffic. These devices each fulfil different purposes and are summarised in Table II. The design of the first generation of Air Quality Monitor (AQM) is presented in [4] with a discussion of improvements for the next version presented in [5]. Both generations of AQMs use the same LoRaWAN hardware which is a Raspberry Pi with a Dragino LoRa Hardware Attached on Top (HAT), as this provides the flexibility and processing power needed to interface with the air quality sensors in use.

To map the network coverage, a node with Global Positioning System (GPS) is used to enable the location of the transmission to be encoded in the message, doing this enables existing web services to be used to plot the data [16]. The location of all transmissions attempts could also be stored locally on the device allowing coverage black spots to be identified, as at the moment the data does not distinguish between no test performed and no coverage. The high power usage/cost of both the Raspberry Pi and Pycom devices means that they are not suitable for long term unattended deployments. To test the reliability of the network, Siconia devices have been deployed in static locations within the covered area and are set to transmit at regular intervals. These devices provide data about the performance of gateways over time and generate datasets for location analysis.

\section{B. Gateways}

The TTN community has around 6000 gateways deployed worldwide, one advantage of LoRaWAN over Sigfox or NB- 


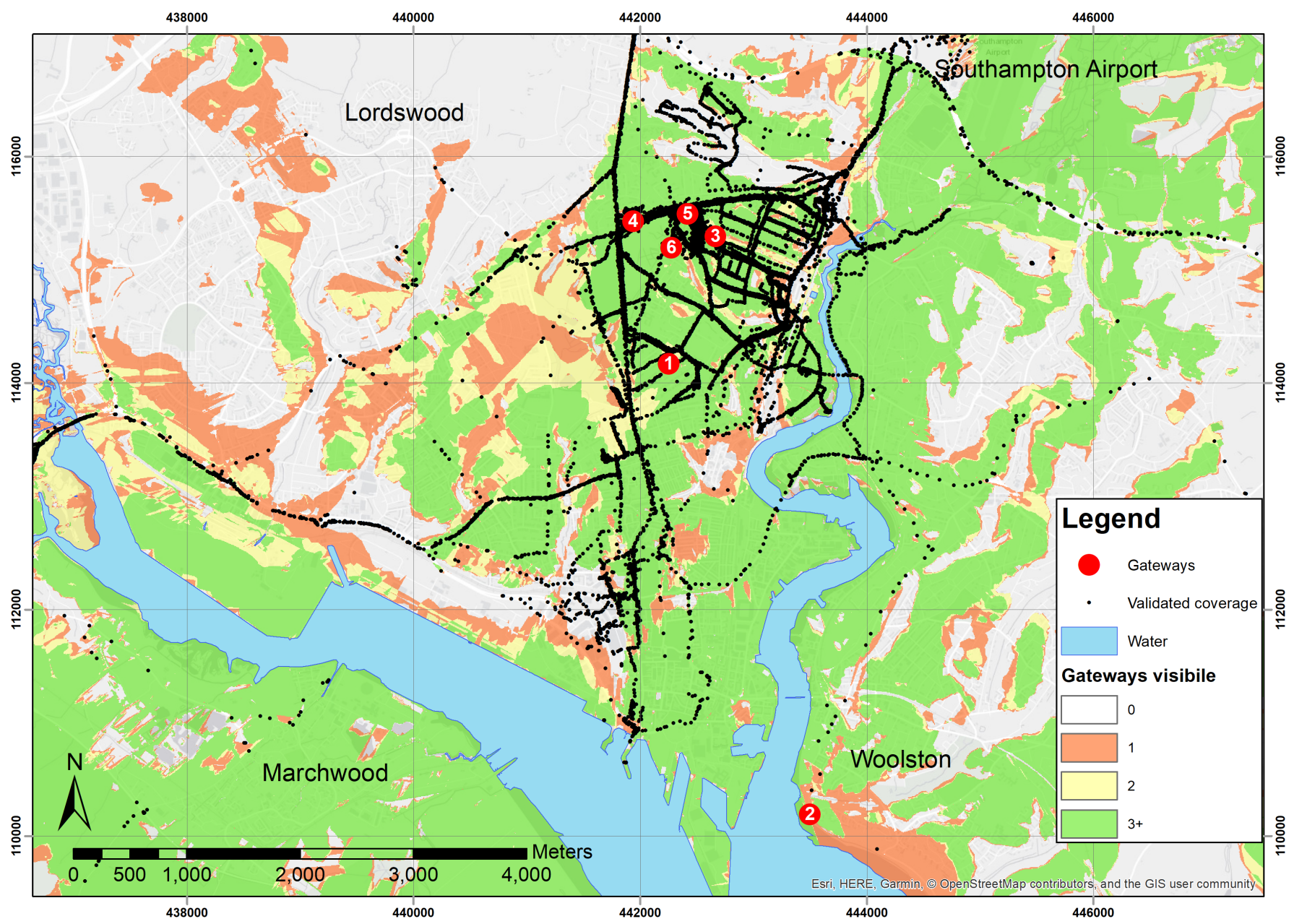

Fig. 1. Map showing deployment area. Approximate line of sight coverage (to point $1 \mathrm{~m}$ above ground level) is shown colour coded by number of gateways available. Points show locations of confirmed coverage, some of which extend beyond line of sight. Map created using data from [16], [17]

IoT is that anyone can deploy a gateway. The availability of gateways has increased dramatically in the last couple of years. There are commercial grade gateways available, which are extremely rugged, reliable and have more advanced hardware. These features come with a high price, and low customisability. Initially the only other option for gateway construction was to construct your own using a LoRa interface and a Single Board Computer (SBC) [19]. Differences between the different gateway construction are shown in Table III. The recently released Things Indoor Gateway [20] significantly reduces the financial outlay required to setup a gateway suitable for personal use, which will encourage further uptake of LoRaWAN. The price point of gateways suitable for larger scale deployments means that building a custom gateway is still a cost-effective proposal.

When constructing a gateway from parts the key consideration is the LoRa hardware interface to use. A lower option is to use the same single channel LoRa HAT as used in the end nodes previously discussed. While single channel operation is perfect for an end device, it is not ideal for a gateway. LoRa supports eight separate frequencies for end nodes to transmit on (region specific). For these channels to be usable, a gateway has to be listening on the specified frequency. With single channel gateways, either one channel has to be used and the others ignored, or the frequency has to be changed at set intervals. Either option means that some traffic will be lost. This limitation means that this approach is only suitable for very low usage controlled scenarios, for example bench testing new devices. Although Things Indoor Gateway available for 69USD removes even this use case. An alternative is to use a multichannel concentrator board which can listen on multiple channels simultaneously. One such board is the iMST iC880A, which has been used for the development of the gateways in Section IV-C. The main advantages of these home built gateways are: price and flexibility - the ability to design the system connectivity and enclosure to best meet your requirements. The lower price point limits the possibility of including more advanced features.

The default for all LoRaWAN devices is to operate in class A, meaning that data can only be received by the end device in a short window after transmission. If the gateway has GPS receiver then it can be used to provide a beacon 
TABLE II

COMPARISON OF DIFFERENT LORAWAN NODES. PRICES CORRECT AS OF JANUARY 2019, PRICE IS FOR WORKING HARDWARE EXCLUDING A POWER SUPPLY, WHEN NOT INCLUDED.

\begin{tabular}{lccc} 
& Raspberry Pi \& LoRa HAT & Siconia & Pytrack \& LoPy \\
\hline Manufacturer & Raspberry Pi \& Seeed Studio & Sagemcom & Pycom \\
LoRa hardware & Dragino LoRa & Proprietary & Proprietary \\
Order of power usage & W & mW & mW \\
Built in GPS & Yes & No & Yes \\
Customisability & High & None & Medium \\
Battery Included & No & Yes & No \\
Programming Language & Various & JavaScript & MicroPython \\
Enclosure type & None supplied & 40 & None supplied \\
Cost (USD) & 100 & & 115
\end{tabular}

TABLE III

COMPARISON OF DIFFERENT LORAWAN GATEWAY SOLUTIONS. PRICES CORRECT AS OF JANUARY 2019 AND EXCLUDE SUITABLE EXTERNAL ANTENNA, MOUNTING HARDWARE, AND POWER SUPPLIES.

\begin{tabular}{|c|c|c|c|c|c|}
\hline & $\begin{array}{c}\text { Raspberry Pi } \\
\text { Single channel-gateway }\end{array}$ & $\begin{array}{c}\text { Raspberry Pi } \\
\text { Multi-channel-gateway }\end{array}$ & $\begin{array}{c}\text { The Things Indoor } \\
\text { Gateway }\end{array}$ & The Things Gateway & Kerlink iBST \\
\hline LoRa hardware & Dragino LoRa & IMST iC880A & Proprietary & Proprietary & Proprietary \\
\hline $\begin{array}{l}\text { Simultaneous } \\
\text { channels }\end{array}$ & 1 & 8 & 8 & 8 & $\leq 16$ \\
\hline Price (USD) & 100 & 250 & 68 & 400 & 2500 \\
\hline Deployment scale & Desk & Campus & Desk & Campus & County \\
\hline Uplink & WiFi/Ethernet & WiFi/Ethernet & WiFi & WiFi/Ethernet & Ethernet / GPRS \\
\hline Accurate timestamp & No & No & No & No & Yes \\
\hline Waterproof enclosure & No & No & No & No & Yes \\
\hline
\end{tabular}

broadcast which enables accurate time alignment between end devices thus enabling class $\mathrm{B}$ which includes scheduled receive windows [9]. Modes other than class A require the full software and hardware stacks to be compatible with the mode. Software supporting this is not currently available.

As discussed in Section II multilateration can be used to locate end nodes. The accuracy of the calculated location is dependent on the gateway hardware in use, the number of gateways that receive the message and the type of the gateway that receives the transmission. All gateways can be used to provide signal strength measurements which can be used for RSSI-based location calculations. For TDOA localisation calculations an accurate time stamp is needed for the message. This can be generated on the Kerlink iBTS gateways as they are fitted with hardware optimised for this. This data can then be fed into the Collos location service [21] which uses this data to calculate the best position fix. RSSI gives accuracy of $1000 \mathrm{~m}$ to $2000 \mathrm{~m}$ compared to TDOA which is in the range $20 \mathrm{~m}$ to $200 \mathrm{~m}$ [22]. An evaluation of LoRaWAN localisation is presented by Fargas et al. [23].

For the Southampton deployment different gateway types are used: Kerlink iBST stations to enable the performing of localisation experiments and multi-channel Raspberry $\mathrm{Pi}$ gateways to fill in coverage and enable gathering additional statistics. This has enabled the required features to be deployed across the city and to perform comparisons of the different gateways. The gateway locations used are shown in Figure 1.

\section{Raspberry Pi gateway development}

Multiple versions of the custom gateway deployed. All versions make use of the IMST iC880A LoRaWAN concentrator. This board enables simultaneous listening to $8 \mathrm{LoRa}$ channels. The iC880A provides a Serial Peripheral Interface (SPI) interface to the radio module. This interface is spread across 26 pins on the Printed Circuit Board (PCB). This form factor needs adapting before it can be interfaced with the SBC used to provide the processing and network connection for the gateway. The earliest versions of the gateway used jumper wires to connect the concentrator to the SBC, an alternative version at the same time used a custom made strip board adaptor. The strip board deployment, while in theory stronger and more robust failed due to corrosion, the jumper wires were reliable until they were replaced as part of a scheduled upgrade. Other deployments are still successfully running using the jumper wire approach [19].

As an alternative to the strip board an off-the-shelf PCB was purchased. This PCB was designed to provide an interface between the iC880A and a Raspberry Pi [24]. As well as interfacing between the concentrator and SBC, it also offers connections for other hardware, including a temperature/humidity sensor. This PCB provided a convenient and robust mounting solution, but did not have the required connections for the GPS testing proposed.

To address this issue a new PCB was developed, the Pi-CoT. The Pi-CoT provides the same functionality as the previous PCB and adds: a Real Time Clock (RTC), Electronically Erasable Programmable Read-Only Memory (EEPROM) for HAT identification, mounting for an Adafruit Ultimate GPS module, mounting for an MT3339 GPS receiver, and connections for serial and Pulse Per Second (PPS) from the GPS receivers. This enables accurate time keeping on the Raspberry $\mathrm{Pi}$, and additional functionality on the LoRaWAN gateway. An example of the Pi-CoT PCB is shown in Figure 2. The 
GPS module used provides an accurate time-pulse for the LoRaWAN concentrator therefore enabling class B operation.

The iC880A PCB offers space for a ublox GPS module to be retro-fitted to the board. However, having fitted the required components, mistakes have been identified on the PCB. These mistakes combined with the difficulty of the modification required mean it is not recommended [25].

The deployment of gateway for any wireless communication protocol requires protection of the electronics. The gateway deployed use an off-the-shelf outdoor enclosure. This enclosure is pre-designed to accommodate up to ten $\mathrm{N}$-connectors, and three cable glands. This provides a waterproof enclosure for the equipment. To reduce the complexity of deployment Power over Ethernet (PoE) is used to provide power to the gateway. This reduces the number of cables required, and all external cabling is low-voltage. The Kerlink iBST gateway can accept PoE directly, but this is not the case for Raspberry $\mathrm{Pi}$ or other SBC based gateways. So a PoE splitter is also contained in the enclosure, which provides $5 \mathrm{~V}$ and network connections for the SBC. To protect the equipment, lightning arresters are included on both the antenna connection and the network cable. A complete custom-built gateway is shown in Figure 2. This shows the latest version of the multi-channel Raspberry Pi gateways used in this deployment.

\section{Southampton Gateway DEPLOYMENT}

When finding a new gateway location, there are two approaches to deciding on a location. The first is to work out where would provide the best coverage and then work out the logistical and permission challenges. The second approach is to identify locations for which permission can easily be obtained and are suitable from a logistical point of view and deploy gateways on these sites. The gateway locations used in this deployment have been obtained using a hybrid approach, in which the available locations are evaluated and the best locations chosen. This approach has led to a dense deployment of gateways around the University campus with 4 locations (36) within a $\mathrm{km}^{2}$, other gateways are located at a residential building (1) and a sailing club (2) shown in Figure 1. Locations 3 and 4 have both custom built and commercial gateways, locations 2 and 5 are commercial gateways, and locations 1 and 5 are custom gateways. All gateways are deployed at roof height with external antennas.

These locations give a reasonable coverage of the city, with $64 \%$ of the area shown in Figure 1 within line of sight of at least one gateway. For a reliable citywide coverage ideally all areas should be within range of at least two gateways (54\%), this means that a single gateway outage does not cause black spots in the coverage. To calculate the location of a transmission, at least three gateways need to receive the signal, the area $(46 \%)$ where three or more gateways have line of sight is shown in green on Figure 1, this does not guarantee the calculation of an accurate position as the spread of the gateways is a major factor on the quality of the position fix. Figure 1 also shows the points where LoRaWAN messages have been sent and confirmed to be received using

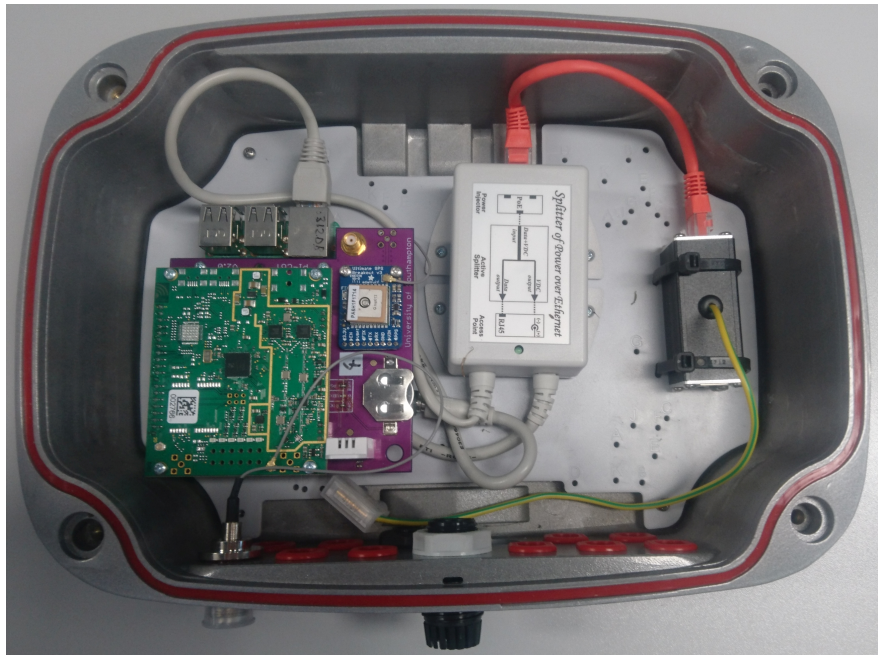

Fig. 2. Custom built LoRaWAN gateway based around a Raspberry Pi 3 Model B, and an iMST iC880A. The purple circuit board was designed for this use case and is available from [26]. All other components are available off-the-shelf, with the backplate being adapted to suit the hardware in use. Power is provided using Power over Ethernet (PoE).

the TTNMapper [16] service. These points show that coverage is not strictly limited to line of sight, but it can provide an indication of the coverage. The work to evaluate and improve the coverage is on-going as additional gateway locations and data become available.

All the gateways that we have deployed around Southampton forward their data to TTN rather than using a proprietary network because the advantages of being able to leverage the community services such as Collos and TTNMapper which are built on top of TTN currently outweigh the limitations. One of the disadvantages of using off-the-shelf gateways is lack of configuration flexibility, this has meant that it is not possible to record detailed usage statistics for the gateways. A configuration error meant that no data was collected for location 1. During 2018, the custom built gateway at location 3 has usage data available for 313 days and received an average of 1274 messages per day. The custom built gateway at location 4 has usage data available for 195 days and an average of 1353 messages per day. The gateway at location 6 received an average of 165 messages per day for the 35 days for which data is available. This data is now being gathered for all custom gateways in the deployment which will enable more detailed analysis to be performed.

\section{CONCLUSIONS AND FUTURE WORK}

LoRaWAN has shown to be a useful communication medium for IoT deployments in a city environment. The flexibility of the custom made gateways and the lower price point has enabled more gateways to be deployed than would otherwise have been possible. The effort required to build and test these custom gateways means that when scaling beyond city scale the off-the-shelf gateway such as the Kerlink iBTS is the most suitable option. 
The use of custom made Raspberry Pi gateways using the iC880A has required the development of a new PCB which has been made freely available to the community [26]. This PCB has additional features which provide the required hardware for the operation of class B LoRaWAN devices.

The long term future of LoRaWAN is not guaranteed, it is the only LPWAN for which is possible to easily deploy your own gateways. The roll of of $5 \mathrm{G}$ mobile infrastructure and future standardisation means that other alternatives such as NB-IoT may be able to offer ubiquitous coverage will therefore be a strong competitor. Switching from deploying your own network to using national infrastructure changes the cost mode. Deploying your own network has a high upfront cost, but minimal ongoing costs. Using a national infrastructure has no or very low up front costs, but a subscription and/or a permessage fee may be charged.

A significant community has been built around the use of LoRaWAN which will be disrupted by the move to carrier based system. The LoRaWAN community built around TTN offers valuable advice tutorials as well as an annual conference focused on new developments in LoRaWAN and interesting use cases of the technology.

This research shows that despite not using optimal gateway locations due to access constraints good coverage city scale LoRaWAN coverage can be achieved. This coverage has been provided by using rooftop locations for which access is available. This deployment is now generating datasets which can be analysed to evaluate the performance and utilisation of the gateways. Localisation data is being gathered which will enable the accuracy of the multilateration based location calculation to be evaluated. Now that the infrastructure has been installed the value of additional use cases will be investigate. Potential uses include: bin usage, parking space occupancy, car counting, and asset tracking.

Dataset available from doi.org/10.5281/zenodo.2542880.

\section{ACKNOWLEDGEMENTS}

We acknowledge funding from the following sources: Engineering and Physical Sciences Research Council [EP/P004024/1 and EP/M508147/1]; Next Generation Unmanned Systems Science Centre for Doctoral Training supported by the Natural Environmental Research Council [NE/L002531/1]; the Leverhulme Trust through the Southampton Marine and Maritime Institute; University of Southampton ADR fund 2018; Richard Lansdowne and Semtech for their ongoing LoRaWAN support. The New Forest Park Authority, Forestry England, and Southampton Sailing Club for hosting LoRaWAN gateways. Josh Curry and Nick Harris for installing and providing data for their gateway (location 6 in Figure 1).

\section{REFERENCES}

[1] A. Bertolaud, J. Delclef, V. Delport et al., LoRaWAN 1.1 Specification, N. Sornin, Ed. LoRa Alliance, 2017, no. 1.1.

[2] J. C. Zuniga and B. Ponsard, "Sigfox System Description," in IETF 97. Seoul, South Korea: IETF, 2016, p. 9.
[3] R. Ratasuk, B. Vejlgaard, N. Mangalvedhe, and A. Ghosh, "NB-IoT system for M2M communication," in 2016 IEEE Wireless Communications and Networking Conference Workshops, WCNCW 2016, no. Wd5g. Doha, Qatar: IEEE, 2016, pp. 428-432.

[4] S. J. Johnston, P. J. Basford, F. M. J. Bulot et al., "IoT deployment for city scale air quality monitoring with Low Power Wide Area Networks," in In Proceedings of the Global IoT Summit 2018, Bilbao, Spain, 4-7 June 2018, May 2018.

[5] S. Johnston, P. Basford, F. Bulot et al., "City Scale Particulate Matter Monitoring Using LoRaWAN Based Air Quality IoT Devices," Sensors, vol. 19, no. 1, p. 209, jan 2019.

[6] K. Mekki, E. Bajic, F. Chaxel, and F. Meyer, "A comparative study of LPWAN technologies for large-scale IoT deployment," ICT Express, jan 2018.

[7] J. P. Bardyn, T. Melly, O. Seller, and N. Sornin, "IoT: The era of LPWAN is starting now," European Solid-State Circuits Conference, vol. 2016October, pp. 25-30, 2016.

[8] X. Yang, E. Karampatzakis, C. Doerr, and F. Kuipers, "Security vulnerabilities in LoRaWAN," in Proceedings of ACM/IEEE International Conference on Internet of Things Design and Implementation, IoTDI 2018. Orlando, Florida, USA: IEEE, 2018, pp. 129-140.

[9] I. Butun, N. Pereira, and M. Gidlund, "Analysis of LoRaWAN v1.1 security," in Proceedings of the 4th ACM MobiHoc Workshop on Experiences with the Design and Implementation of Smart Objects SMARTOBJECTS '18. New York, New York, USA: ACM Press, 2018, pp. 1-6.

[10] S. E. W. Bush, "Sigfox adds a basestation and geolocation," 2018. [Online]. Available: https://www.electronicsweekly.com/news/products/ sigfox-adds-basestation-geolocation-2018-10/

[11] “The Things Network," 2018. [Online]. Available: https://www. thethingsnetwork.org/

[12] H. Liu, H. Darabi, P. Banerjee, and J. Liu, "Survey of wireless indoor positioning techniques and systems," IEEE Transactions on Systems, Man, and Cybernetics, Part C (Applications and Reviews), vol. 37, no. 6, pp. 1067-1080, 2007.

[13] W. Guibene, J. Nowack, N. Chalikias et al., "Evaluation of LPWAN Technologies for Smart Cities: River Monitoring Use-Case," 2017 IEEE Wireless Communications and Networking Conference Workshops, WCNCW 2017, pp. 1-5, 2017.

[14] P. J. Landrigan, R. Fuller, N. J. Acosta et al., "The Lancet Commission on pollution and health," October 2017.

[15] N. Park, "Dataset: Estimates of the population for the uk, england and wales, scotland and northern ireland," Office for National Statistics, 2018.

[16] J. Meijers, "TTNMapper," 2019. [Online]. Available: https://ttnmapper. org/

[17] EDINA Digimap Ordnance Survey Service, "OS Terrain 5 [ASC geospatial data], Scale 1:10000," 2018. [Online]. Available: https: //digimap.edina.ac.uk

[18] "Lacuna space," 2019. [Online]. Available: http://lacuna.space

[19] N. R. Harris and J. S. Curry, "Development and range testing of a LoRaWAN system in an urban environment," International Journal of Electronics and Communication Engineering, vol. 12, no. 1, pp. 47-55, 2018.

[20] K. I. Easwaran and K. I. Easwaran, "The things indoor gateway," 2019. [Online]. Available: https://www.thethingsnetwork.org/docs/gateways/ thethingsindoor/

[21] Semtec, "Collos: Collaborative location service," 2017. [Online]. Available: http://preview.collos.org/

[22] LoRa Alliance, "LoRaWAN Geolocation Whitepaper," 2018. [Online]. Available: https://docs.wixstatic.com/ugd/eccc1a d43b3b29dfff4ec2b00f349ced4225c4.pdf

[23] B. C. Fargas and M. N. Petersen, "GPS-free geolocation using LoRa in low-power WANs," in Global Internet of Things Summit (GIoTS), 2017. IEEE, 2017, pp. 1-6.

[24] C. Hallard, "Raspberry pi iC880A and linklab lora gateway shield," 2017. [Online]. Available: https://pcbs.io/share/rmVdD

[25] P. J. Basford, "Adding a GPS receiver to the iC880A LoRaWAN concentrator," 2017. [Online]. Available: https://computenodes.net/2017/ 09/05/adding-a-gps-receiver-to-the-ic880a-lorawan-concentrator/

[26] P. J. Basford, S. J. Johnston, and S. J. Cox, "computenodes/pi-cot: Pi Concentrator on Top V2.0.1 release," 2019. [Online]. Available: doi.org/10.5281/zenodo.2539362 